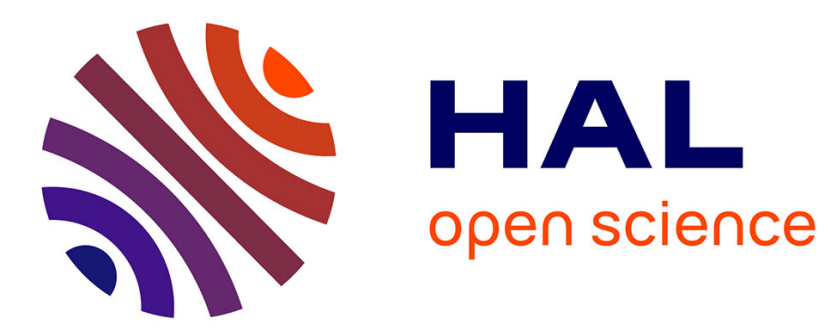

\title{
L'enseignement de la nation dans l'éducation en France: objectifs et questionnements
}

Gérard-François Dumont

\section{To cite this version:}

Gérard-François Dumont. L'enseignement de la nation dans l'éducation en France: objectifs et questionnements. Outre-terre. Revue européenne de géopolitique, 2005, 13, pp.393-422. 10.3917/oute.012.0393 . halshs-00769654

\section{HAL Id: halshs-00769654 \\ https://shs.hal.science/halshs-00769654}

Submitted on 2 Jan 2013

HAL is a multi-disciplinary open access archive for the deposit and dissemination of scientific research documents, whether they are published or not. The documents may come from teaching and research institutions in France or abroad, or from public or private research centers.
L'archive ouverte pluridisciplinaire HAL, est destinée au dépôt et à la diffusion de documents scientifiques de niveau recherche, publiés ou non, émanant des établissements d'enseignement et de recherche français ou étrangers, des laboratoires publics ou privés. 


\title{
L'enseignement de la nation dans l'éducation en France : objectifs et questionnements
}

\author{
Gérard-François Dumont
}

Dans la formule « enseigner la nation » figurent deux termes qui paraissent d'une acception simple. La nation se définit, d'un point de vue géographique et institutionnel, comme un territoire organisé sous une forme étatique, ce qui lui vaut d'appartenir à l'Organisation des Nations unies. Enseigner consiste à transmettre des méthodes et des connaissances à d'autres personnes.

Mais derrière ces deux définitions simples se cache une grande complexité. Si enseigner est un comportement aisé à comprendre, il est plus délicat d'en analyser le contenu : on peut en effet enseigner le pire et le meilleur, des méthodes pour bonifier une terre afin qu'elle permette des productions vivrières de qualité ou la technique pour préparer des actes terroristes, comme dans les manuels trouvés en Afghanistan après le 11 septembre 2001.

Résumer, selon la définition ci-dessus, la nation à un territoire dont la perception, tout particulièrement celle de ses frontières, est aisée à discerner grâce à une carte, n'offre pas de difficulté particulière. Quant aux zones maritimes des nations, elles sont précisées en application du droit international, ce qui n'empêche pas, ici ou là, la persistance de conflits. En outre, lorsque plusieurs pays revendiquent le même territoire, les cartes nationales peuvent différer. Néanmoins, aujourd'hui, la plupart des revendications territoriales ne concernent que des terres situées à la marge des frontières nationales, par exemple des îles.

Dès que l'on quitte le sens factuel donné à la nation, caractérisée par un territoire, par une population qui y réside ou qui y jouit de la nationalité juridique, par une constitution qui lui est propre, la définition du mot « nation » devient

Gérard-François Dumont, recteur, professeur à l'université de Paris-Sorbonne, président de Population \& Avenir. 
délicate et peut reposer sur différents concepts. La nation peut alors correspondre, selon une deuxième définition, à un ensemble d'hommes auxquels on suppose une origine commune ou, selon une troisième définition, à un groupe humain conscient de son unité ${ }^{1}$. Ces deux dernières définitions se réfèrent alors à des questions de nature historique, sociale et culturelle pouvant donner lieu à de multiples interprétations, voire à des utilisations idéologiques. Il suffit pour cela de rappeler la nécessaire distinction entre sentiment national et nationalisme. Ce dernier mot prend d'ailleurs plusieurs sens selon qu'il s'agit de revendiquer le droit de former une nation contre une puissance extérieure, d'exalter le sentiment national au point d'engendrer la xénophobie ou des démarches de fermeture et d'isolement, ou encore d'élaborer une doctrine fondée sur l'idée de supériorité et subordonnant en conséquence toute politique au développement de la puissance nationale, jusqu'à revendiquer sa puissance à l'extérieur sans limitation de souveraineté.

Outre le nationalisme, qui peut prendre ces trois sens différents, la déclinaison du mot «nation » peut conduire à l'emploi du mot « nationalité », comme en Espagne, où l'on distingue la nation (Espagne espagnole) des nationalités (basque, catalane...).

Pour notre part, nous définirons ci-après la nation comme projet remplissant deux missions essentielles : « La nation est un projet incitant les habitants qui vivent sur un territoire commun à placer au-dessus de leurs intérêts particuliers le sens du bien commun afin de remplir deux missions : empêcher sa destruction par elle-même et par d'autres, c'est-à-dire assumer la cohésion à l'intérieur et la sécurité extérieure, la concorde sociale et la paix avec les autres ${ }^{2}$. »

La question sémantique ainsi précisée, il convient d'abord d'examiner le contexte et l'évolution de l'enseignement de la nation en France. Nous étudierons ensuite cet enseignement tel qu'il figure dans les programmes actuels. Enfin, nous verrons que cette question de l'enseignement de la nation se trouve confrontée, dans cette période du milieu des années 2000, à de nombreux questionnements.

1. En outre, les données historiques font que les nations sont soumises au vent de l'histoire : certaines peuvent disparaître, d'autres peuvent naître, comme la nation américaine.

2. Cf. Gérard-François Dumont, «La nation, l'identité et le droit à l'indifférence », Géographie et cultures, $\mathrm{n}^{\circ} 42$, été 2002. 


\section{Le contexte et l'évolution de l'enseignement de la nation en France depuis deux siècles}

Le souci d'enseigner la nation en France, qui explique certainement la dénomination du ministère de l'Éducation, ne fait pas de doute. Néanmoins se pose la question de son contenu et de sa place, qui évoluent au fil du temps.

\section{La France, l'un des rares pays où l'éducation se veut «nationale »}

La volonté présente en France, en 2005, d'enseigner la nation résulte d'une longue histoire. Elle s'inscrit dans le cadre fixé par l'idée selon laquelle il revient à l'État de prendre en main les affaires d'enseignement parce que cellesci doivent relever non pas d'institutions privées, religieuses ou laïques, mais des pouvoirs publics. Aussi l'expression « éducation nationale » remonte-t-elle à la fin du XVIII ${ }^{e}$ siècle, où elle était employée par ceux qui appelaient l'État à régler les questions d'enseignement.

Néanmoins, à la Révolution, bien que la formule ne soit pas institutionnalisée, le caractère national, au sens géographique du terme, de l'éducation se met en place. La Révolution crée toutes sortes d'établissements d'enseignement, secondaires ou supérieurs, afin de doter la République d'un réseau répandant l'instruction dans toutes les classes de la société.

Sous l'Empire, l'État considère que l'existence d'un réseau d'établissements ne suffit pas à assurer la formation des cadres du pays et qu'il faut une structure forte, stable et centralisée pour orienter le fonctionnement du système. Dans cette perspective, l'Université impériale est fondée par la loi du 10 mai 1806, puis organisée par le décret du 17 mars 1808. Plus qu'une administration, c'est une sorte de corporation laïque qui jouit d'une large autonomie par rapport aux autres services de l'État. À sa tête se trouve le Grand-Maître de l'Université, nommé par l'Empereur et lui rendant compte directement. Ce Grand-Maître partage le pouvoir avec le Conseil de l'Université, formé de membres du « corps enseignant », expression qui apparaît à cette époque.

Pendant quelque temps, la Restauration envisage de démanteler ce système, qui lui paraît trop indépendant du trône comme de l'autel. En 1815, une Commission de l'Instruction publique, placée sous l'autorité du ministre de l'Intérieur, est donc nommée pour remplacer le Grand-Maître. Mais elle maintient pour l'essentiel les prérogatives de l'Université, ce qui se traduit bientôt par sa transformation en Conseil royal de l'Instruction publique (1820), puis par le rétablissement du titre de Grand-Maître (1822). Au fil des décennies, diverses décisions interviennent, dont la loi Guizot de 1833 sur les écoles primaires, la création du baccalauréat en 1840, la loi Falloux de 1850, la création des académies avec un recteur à leur tête en 1854, ou la loi Ferry de 1882. 
Plus tard, dans les années 1910-1920, l'expression «éducation nationale » réapparaît sous la plume des adversaires de la division du système éducatif en filières distinctes et, en 1932, le gouvernement d'Édouard Herriot décide de dénommer l'Instruction publique «Éducation nationale ». Anatole de Monzie, premier ministre à porter cette nouvelle titulature, explique dès sa prise de fonction qu'elle est synonyme d'égalité scolaire et de développement de la gratuité et que, en somme, qui dit « éducation nationale » dit « tronc commun ».

Après 1932, cette titulature demeure mais est remise en cause par deux fois. D'abord pendant les premiers mois du gouvernement de Vichy, où l' « Instruction publique » fait sa réapparition. Mais, dès le 23 février 1941, l'appellation « Éducation nationale » est rétablie. Elle est parallèlement conservée à Londres, puis à Alger, par les gouvernements du général de Gaulle. Sous la IV ${ }^{\mathrm{e}} \mathrm{Répu}-$ blique, puis sous les présidences du général de Gaulle et de Georges Pompidou, aucun changement n'intervient.

Pendant son septennat, Valéry Giscard d'Estaing innove en privant le ministère de l'Éducation de son épithète de «nationale ${ }^{3}$ ». Mais, le 22 mai 1981, elle revient dès le premier gouvernement de Pierre Mauroy, avec Alain Savary.

Avec cet adjectif «national » accolé à l'intitulé du ministère de l'Éducation, la France se présente comme un cas singulier en Europe, comme l'attestent les dénominations des ministres membres du Conseil européen Éducation, Jeunesse, Culture et Audiovisuel de l'Union européenne. En effet, parmi ses vingt-cinq membres, outre le ministre français, seuls trois ont l'adjectif « national ».

La Grèce compte un « ministre de l'Éducation nationale et des Affaires religieuses », intitulé qui dénote une différence majeure dans la conception de l'« éducation nationale » en France et en Grèce. En effet, quel violent débat s'instaurerait en France si les Affaires religieuses étaient associées à l'Éducation et non plus à l'Intérieur ! Le Luxembourg, pays où le tiers de la population est de nationalité étrangère et où plus de la moitié de la population active ayant un emploi est également de nationalité étrangère, compte un «ministre de l'Éducation nationale et de la Formation professionnelle ». Enfin, en Pologne, l'intitulé «ministre de l'Éducation nationale et des Sports » se comprend mieux lorsqu'on se rappelle les vicissitudes que l'histoire a fait subir à la nation polonaise

3. Et surtout en confiant tout ce qui relève de l'enseignement supérieur à un secrétariat d'État, puis à un ministère autonome. Initiative appelée d'ailleurs à un certain avenir puisqu'elle s'est maintenue presque sans interruption jusqu'en 1995, sous des appellations diverses («Universités » de 1974 à 1986, « Enseignement supérieur » de 1986 à nos jours) et avec des statuts divers (secrétariat d'État de 1974 à 1978, de 1983 à 1986, et en 1995 dans le gouvernement d'Alain Juppé ; ministère délégué de 1986 à 1988 ; ministère de 1978 à 1981 et de 1993 à 1995). 
avec par exemple, dans la première moitié des années 1940, la violence hitlérienne visant à faire table rase de toute identité et de toute culture polonaises. Dans les vingt et un autres pays de l'Union européenne de 2005, l'Éducation est une charge ministérielle qu'aucun adjectif ne définit. Il en est de même dans les deux pays qui doivent rejoindre l'Union en 2007, la Roumanie et la Bulgarie.

Outre la désignation du ministère chargé de l'Éducation, et sans doute en conséquence de cette désignation, il faut préciser que le souci d'enseigner la nation en France s'accorde avec le caractère national des programmes et des manuels scolaires, qui valent des Caraïbes au Pacifique en passant par l'océan Indien, pour l'élève d'un établissement situé en Alsace comme pour le jeune Réunionnais ou l'adolescent de la Guadeloupe ${ }^{4}$. Enseigner la nation est donc incontestablement une préoccupation en France. Mais cela consiste à enseigner quoi ?

\section{Quelle nation française enseigner? Son territoire? Ses symboles?}

Autrement dit : qu'est-ce que la nation française ? Cette question particulièrement complexe a suscité des débats dès le XVIII ${ }^{\mathrm{e}}$ siècle $^{5}$. Sous la Révolution, Sieyès, influencé par Le Contrat social de Rousseau, voit dans la nation l'association volontaire et contractuelle de citoyens égaux ; c'est un « corps d'associés vivant sous une loi commune et représentés par la même législature ${ }^{6} »$. Au

4. Même si quelques programmes dits « adaptés » se mettent en place depuis la rentrée 2000. Pour la Guadeloupe et la Martinique, ils se composent des programmes nationaux auxquels on ajoute ou substitue partiellement des questions axées sur la Caraïbe, les DFA (départements français d'Amérique) ou l'un des trois départements.

À la suite de la modification en 2004-2005 des programmes de terminale, et pour que les épreuves du baccalauréat puissent porter sur ces questions « adaptées », celles-ci ont été modifiées.

En géographie de seconde, il n'y a aucune adaptation, mais l'enseignant doit prendre quelques exemples concernant la Caraïbe. En histoire de seconde, à la place de l'une des quatre premières questions du programme, l'enseignant traite « La Caraïbe au XVIII ${ }^{\mathrm{e}}$ siècle ». En géographie de première, le programme adapté invite à traiter la région dans laquelle se trouve le lycée. En géographie de terminale, à la place de «La Méditerranée, une interface Nord/Sud », l'enseignant traite « La Caraïbe, une interface Nord/Sud ». En histoire de terminale, en plus du programme, l'enseignant traite «Économie, société et culture en Guadeloupe, en Martinique et/ou en Guyane depuis 1945 ».

En 2000 ont été édités un manuel pour le lycée et un second pour le collège, qui a aussi ses adaptations.

5. Nous utilisons ici l'un des chapitres de l'ouvrage que nous avons dirigé : Ralph Schor, dans Gérard-François Dumont et col., Les racines de l'identité européenne, Paris, Économica, 1999.

6. Sieyès, Qu'est-ce que le tiers état ?, Paris, Éditions des Archives contemporaines, 1985. 
$\mathrm{XIX}^{\mathrm{e}}$ siècle, Augustin Thierry souligne à la fois les origines raciales et la fusion progressive d'éléments d'abord disparates. Michelet minore le critère racial et insiste sur l'interaction de l'homme et du milieu : selon lui, la France est une personne dont les provinces constituent les membres et Paris l'esprit assimilateur. En 1882, Ernest Renan, dans une conférence célèbre, Qu'est-ce qu'une nation ?, répond : "C'est une âme, un principe spirituel [...], la possession en commun d'un riche legs de souvenirs [...], le consentement actuel, le désir de vivre ensemble [...]. Avoir souffert, joui, espéré ensemble, voilà qui vaut mieux que des douanes communes et des frontières conformes aux idées stratégiques [...]. L'existence d'une nation est un plébiscite de tous les jours. » Quant à Maurice Barrès, à la fin du XIX ${ }^{\mathrm{e}}$ siècle, il célèbre le culte de la terre natale et des ancêtres qui ont bâti la France.

$\mathrm{Au}$ début du $\mathrm{XX}^{\mathrm{e}}$ siècle, le géographe Paul Vidal de La Blache ne s'éloigne guère de Barrès : pour lui, l'identité est à rechercher dans les campagnes et dans l'adaptation des hommes au sol sur lequel ils ont vécu durant des siècles. André Siegfried, fondateur de la science politique en France, et d'autres érudits comme Élie Faure traquent, avec les méthodes de la psychologie sociale, les éléments permanents d'un tempérament national.

À la lecture des écrits des auteurs du passé, l'identité nationale juxtapose donc des faits vérifiables et des conceptions dont la subjectivité apparaît plus ou moins forte. Ainsi, une recherche historique sur cette identité revient à mettre en valeur les traces du passé dans la France d'aujourd'hui, traces objectives ou non. En 1989, Fernand Braudel se fixe un tel programme dans L'Identité de la France : «Alors qu'entendre par identité de la France ? Le résultat vivant de ce que l'interminable passé a déposé patiemment par couches successives [...]. En somme un résidu, un amalgame, des additions, des mélanges [...]. Se reconnaître à mille tests, croyances, discours, alibis, vaste inconscient sans rivages, obscures confluences, idéologies, mythes, fantasmes ${ }^{7}$. 》

\section{L'addition de l'histoire et de la géographie}

\section{au service de l'enseignement de la nation}

Néanmoins, en dépit des difficultés à sérier la définition de la nation et alors que l'intitulé ministériel est «Instruction publique » et non encore « Éducation nationale ", dès le XIX ${ }^{\mathrm{e}}$ siècle, l'école devient un instrument privilégié pour développer le sentiment d'appartenance nationale des futurs citoyens. L'enseignement de la géographie, de l'histoire et de l'éducation civique est mis au service de l'État pour construire cette identité. Une fonction majeure est assi-

7. Cf. Fernand Braudel, L'Identité de la France, Paris, Flammarion, 1989. 
gnée à ces trois disciplines : construire un socle commun de référents et de valeurs pour véhiculer un ensemble de certitudes formant une identité, voire une idéologie nationale.

Dans la France de la III ${ }^{e}$ République, le Tableau de la géographie de la France de Vidal de La Blache et l'Histoire de France de Lavisse constituent les fondements de l'enseignement patriote et laïc de l'école républicaine. L'enseignement de la nation résulte donc principalement d'une addition de l'histoire et de la géographie. Il se conforme à Michelet, pour qui « l'histoire est d'abord toute géographie ${ }^{8}$ ». Michelet pense que «le sol n'est pas, pour les sociétés humaines, un simple parterre immobile, un inerte plancher de théâtre ${ }^{9}{ }$. Cette approche se retrouve chez Lucien Febvre, qui, en 1922, dans l'introduction à La Terre et l'évolution humaine, pose le « problème des influences géographiques » dans l'histoire des hommes.

Dans l'examen de la genèse du sentiment national, l'attachement naturel au sol natal est très présent. Pour le réactionnaire Maurice Barrès, cet enracinement constitue un facteur identitaire décisif. Plus tard, le communiste Louis Aragon exprime une opinion voisine :

« Je vous salue ma France où les vents se calmèrent !

«Ma France de toujours que la géographie

«Ouvre comme une paume aux souffles de la mer

« Pour que l'oiseau du large y vienne et se confie. »

Jusqu'au milieu du $\mathrm{Xx}^{\mathrm{e}}$ siècle, les écrivains et les géographes enseignent qu'il faut aimer la France, car elle est belle. Ils évoquent en effet la perfection de sa forme hexagonale, l'harmonieuse diversité de ses paysages, l'égale proportion des plaines et des montagnes, l'emplacement heureusement excentré des chaînes les plus hautes, la situation idéale au cœur de la zone tempérée, la modération des climats, les influences physiques et culturelles complémentaires apportées par les frontières continentales et les mers bordières, l'équilibre exceptionnel qui résulte de toutes ces données naturelles. La géographe Jacqueline Beaujeu-Garnier illustre ce point de vue : «Au $37^{\mathrm{e}}$ rang par sa surface, au $13^{\mathrm{e}}$ rang par le nombre de ses habitants, la France tient encore aujourd'hui un rôle de premier plan dans la marche de la civilisation et des événements mondiaux. Sans faire preuve d'un déterminisme abusif, il semble juste de rechercher en partie les causes de cette supériorité dans l'étonnant territoire où

8. Formule figurant dès le début de son Tableau de la France, placé en 1833 en tête du tome II de son Histoire de France.

9. Cf. Lucien Febvre, Lionel Bataillon, La Terre et l'évolution humaine. Introduction géographique à l'histoire (1922), Paris, Albin Michel, 1970, p. 21. 
a pris racine la nation française. Territoire remarquable par sa situation au cœur de l'Europe occidentale, territoire émouvant par la variété de ses paysages et de ses promesses ${ }^{10}$. »

Quant au politologue André Siegfried, il insiste également sur la dimension géographique de l'identité nationale : « La France a trois versants et, du fait de cette triple orientation, elle est à la fois occidentale, continentale, méditerranéenne. Il en résulte un équilibre original et peut-être unique. Par son front atlantique, elle regarde vers le dehors, avec une fenêtre ouverte sur le grand large : elle subit de ce fait des attractions extracontinentales, la tentation des aventures lointaines [...]. En revanche, en tant que continentale, elle tient à l'Europe par un lien de chair impossible à rompre, bien différente en cela de l'insulaire Angleterre [...]. De ce point de vue, nous sommes continentaux, terriens, essentiellement européens [...]. Par son front méditerranéen enfin, la France est en contact immédiat avec l'Afrique, l'Asie, l'Orient, l'ExtrêmeOrient, c'est-à-dire, dans l'espace, avec un monde exotique et prestigieux, et, dans le temps, avec un passé le plus illustre de l'humanité ${ }^{11}$. »

La force des liens qui attachent les Français à leur pays semble d'autant plus déterminante que celui-ci a constitué très précocement son unité géopolitique. L'un des apports décisifs de Michelet est précisément d'avoir mis en évidence la stabilité du cadre étatique dans l'espace français dès le Moyen Âge.

L'enseignement de l'histoire est donc aussi un enjeu important. Néanmoins, à la question « Depuis quand la France ? », la réponse donnée - puisque le choix existe entre « vingt siècles depuis Vercingétorix, quinze siècles depuis Clovis ou onze siècles depuis Charles le Chauve ${ }^{12} »-$ se colore de nuances idéologiques diverses.

En raison de ces réflexions sur l'identité de la nation à enseigner, les élèves de la III ${ }^{\mathrm{e}}$ République sont formés par des maîtres qui leur apprennent l'amour de la patrie, les principes républicains, le culte du progrès, les valeurs morales. Ces thèmes sont popularisés au travers de modèles historiques qui traduisent les qualités essentielles de l'être national : héros mourant pour la France comme le jeune Bara, saints dévoués au pays comme Jeanne d'Arc, chefs disciplinant les énergies à l'instar de Richelieu, savants bienfaiteurs de l'humanité à l'image de Pasteur.

10. Cf. Jacqueline Beaujeu-Garnier, Histoire de France, Paris, Larousse.

11. Cf. André Siegfried, L'Âme des peuples, Paris, Hachette, 1950.

12. Cf. Maurice Agulhon, «La République française : vision d'un historien », dans Paul Isoart, Christian Bidegaray (sous la direction de), Des Républiques françaises, Paris, Économica, 1988, p. 50. 
Outre la géographie et l'histoire, l'enseignement de la nation, sous la III' et la $\mathrm{IV}^{\mathrm{e}}$ République, passe par la langue et la littérature. Dès le premier âge, les enfants vivent dans un univers francophone. La linguistique moderne souligne que le langage est solidaire de la société et la reflète. Or le français, langue de la cour et de l'administration sous l'Ancien Régime, s'est progressivement étendu au détriment des parlers régionaux.

Dans l'enseignement primaire et secondaire, les jeunes reçoivent une formation à peu près identique, définie, comme précisé ci-dessus, par des programmes nationaux. La culture littéraire traditionnelle offre à chacun des références éprouvées : fables de La Fontaine, théâtre du XVII ${ }^{\mathrm{e}}$ siècle, romans du XIX ${ }^{\mathrm{e}}$ siècle, œuvre multiforme de Victor Hugo... L'exercice de la dissertation permet de discipliner les idées et les connaissances. La limpidité d'un raisonnement cartésien est exigée, car, comme l'indique Rivarol, « ce qui n'est pas clair n'est pas français ${ }^{13}$ ». Léopold Senghor a même écrit que le français était la « langue des dieux ${ }^{14} \gg$.

Ainsi le façonnement des jeunes esprits par la géographie, par l'histoire, par la langue et la littérature tient-il une place essentielle dans la formation du lien national. Cette forme d'enseignement dure jusqu'au lendemain de la Seconde Guerre mondiale, et une instruction ministérielle du 4 juillet 1961 rappelle encore que l'éducation civique doit préparer les citoyens au «sacrifice suprême » pour la patrie.

\section{L'effritement de l'enseignement de la nation et le retour de l'histoire nationale}

Dans les années 1960 et 1970, tandis que la géographie évolue vers l'étude de l'espace, multipliant les approches théoriques ou schématiques et s'éloignant assez souvent de la connaissance du territoire, l'histoire s'intéresse de plus en plus à l'économie, à la société, à la vie, au passé des autres pays, et cesse de diffuser le message traditionnel. La nouvelle pédagogie, qui se veut active, fondée sur l'observation, l'enquête, la critique du document, vise à rendre l'élève autonome. La notion d'identité nationale étayée par des repères précis semble se diluer.

En contrepoint, un mouvement d'opinion, qui culmine vers 1979-1980, animé par des historiens et des personnalités de toutes tendances, assure que l'ignorance du passé rend les citoyens amnésiques, incultes et manipulables :

13. Rivarol, De l'universalité de la langue française, 1784.

14. Cf. Léopold Sédar Senghor, Éthiopiques, Paris, Le Seuil, 1956. 
«L'épopée de Jeanne d'Arc n'a plus sa place dans nos programmes... Le long cheminement qui a fait notre patrie n'est jamais présenté à nos élèves. » (Michel Debré, RPR, 3 septembre 1979)

« On tente d'abolir la mémoire de notre peuple. » (Louis Mexandeau, PS, 30 novembre 1979)

«Les pédagogues dissimulent derrière des vues techniques et des concepts à la mode l'impuissance de la nation à définir son destin. » (Maurice Duverger, Le Monde, 25 juin 1980)

À la suite de cette campagne, l'histoire avec faits dominants, dates, personnages, fait son retour à l'école élémentaire. Quelques années plus tard, le 24 octobre 1997, Claude Allègre, ministre de l'Éducation nationale, envisage la création d'un module obligatoire d'éducation à la citoyenneté pour tous les élèves de première et d'une épreuve de morale civique aux concours d'entrée dans les instituts universitaires de formation des maîtres. Le ministre précise : «On ne peut solliciter l'école en laissant chaque enseignant décider de ce qu'est la citoyenneté. Elle est nationale, ses valeurs sont à enseigner partout, et les mêmes. » Claude Allègre veut aussi « rénover l'enseignement de l'histoire » pour que soit expliquée « l'évolution qui a permis petit à petit de construire la République et les trois mots qui forment sa devise. » Il s'agit donc bien de restaurer certaines valeurs enseignées sous la III $^{\mathrm{e}}$ République et considérées comme un lien identitaire puissant.

Après ces hauts et ces bas de l'enseignement de la nation, le souci d'enseigner la nation continue de prévaloir aujourd'hui en France, comme l'attestent les programmes.

\section{L'enseignement de la nation dans les programmes scolaires français}

Nous analyserons tout particulièrement les programmes du primaire, ceux du collège et du lycée entrant davantage dans la réflexion sur le questionnement européen.

L'école primaire et la prise de conscience de l'appartenance à la communauté nationale

Le ministère de l'Éducation nationale a publié en 2004 un livre intitulé Qu'apprend-on à l'école élémentaire? 2004-2005, les programmes ${ }^{15}$. Dans cet

15. Paris, SCÉREN (CNDP)/XO Éditions, 2004. 
ouvrage de 351 pages, qui compte 87000 mots et concerne l'éducation scolaire des enfants de trois à onze ans, le mot «nation », éventuellement sous sa forme adjectivale, apparaît dix fois.

Il est d'abord présent dans la préface du ministre François Fillon, qui précise combien l'idée de communauté nationale doit être assimilée tôt par les enfants de France. Dans un paragraphe intitulé «L'éducation civique et l'apprentissage du "vivre ensemble" », le ministre écrit : « La République a confié à l'École la formation de "ses" citoyens. Cette citoyenneté se prépare dès le plus jeune âge par le biais, notamment, de l'éducation civique. Priorité des programmes, l'éducation civique se constitue progressivement au fil des cycles. Â l'école maternelle, où la majorité des enfants découvre les richesses et les contraintes de l'accueil en milieu collectif, chacun doit apprendre à trouver ses repères et sa place dans le groupe, à coopérer, à comprendre et à s'approprier les règles collectives ; c'est ainsi que se construit une identité d'écolier. À l'école élémentaire, l'élève est appelé à participer pleinement à la vie de l'école ; il commence à dépasser l'horizon de l'école pour prendre conscience de son appartenance à la communauté nationale et saisir la signification de règles de vie collective. Il comprend ce que signifie être citoyen dans sa commune, en France, et il acquiert, au travers de différentes disciplines, des connaissances sur l'État républicain et les valeurs démocratiques, sur l'Europe, la francophonie, le monde ${ }^{16}$. »

Ce paragraphe souligne combien enseigner l'appartenance non à « une », mais à «la » communauté nationale est l'une des missions essentielles de l'école.

Concernant l'école maternelle ${ }^{17}$, le résumé des programmes ne contient ni le terme «nation» ni l'adjectif «national», ce qui peut se comprendre au regard de l'âge des intéressés : entre trois et six ans. L'adjectif «national » apparaît dans le « résumé des programmes de l'école primaire ${ }^{18}$ », sous la rubrique « histoire et géographie », avec la formulation suivante : «Une large place est faite à l'histoire nationale, avec des ouvertures conséquentes sur l'Europe ou sur le monde. La chronologie est respectée, les dates importantes sont apprises, des personnages sont étudiés ${ }^{19}$. $\gg$ Ce passage montre clairement combien l'enseignement de l'histoire doit être au service de la connaissance de la création et de la vie de la nation.

16. Ibid., p. 11.

17. Cf. aussi Qu'apprend-on à l'école maternelle ?, Paris, SCÉREN (CNDP)/Xo Éditions, 2004, qui détaille le cycle des acquisitions premières, de la première section au milieu de la grande section.

18. Qu'apprend-on à l'école élémentaire ?, op. cit., p. 15.

19. Ibid., p. 33. 
Ensuite, le « préambule ${ }^{20}$ », dont il est précisé en note qu'il est « commun aux trois cycles de l'école primaire ${ }^{21} »$, utilise l'adjectif « national » à propos de la langue. Sous la rubrique : «Une culture scolaire partagée ${ }^{22}$ », le français n'est pas seulement désigné comme « une » langue, mais comme « la langue nationale »: " Deux grands axes structurent l'enseignement primaire, la maîtrise du langage et de la langue française, l'éducation civique. Transmettre la langue nationale est l'objectif fondamental ${ }^{23}$. » Le sens de la formulation n'est pas précisé. Cela peut simplement signifier que la langue est nationale dans la mesure où elle doit être commune à l'ensemble des territoires français. Cette qualification peut alors se référer implicitement à la Constitution française, qui, depuis 1992, précise dans son article 2 : «La langue de la République est le français. » Auquel cas la dimension nationale de la langue peut être interprétée de façon simple, en raison de sa nature géographique ou institutionnelle. Rien ne permet de dire s'il s'agit d'un simple véhicule de communication ou d'une langue identitaire, acquis fondamental pour le sentiment national.

Les programmes distinguent ensuite, au sein de l'école élémentaire, le « cycle des apprentissages fondamentaux », embrassant la deuxième moitié de la grande section des maternelles, le cours préparatoire et le cours élémentaire première année, et le " cycle des approfondissements », couvrant le cours élémentaire deuxième année et les deux cours moyens.

\section{Ressentir dès l'âge de neuf ans son appartenance à la nation et connaître ses symboles}

Dans le « cycle des apprentissages fondamentaux ${ }^{24}$ », après le premier point intitulé : « Maîtrise du langage et de la langue française ${ }^{25}$ » et avant le point III : « Mathématiques ${ }^{26}$ », figure une rubrique II : «Vivre ensemble ${ }^{27}$ ». Elle est déclinée comme les autres en trois sous-rubriques : "Objectifs ${ }^{28}$ ", «Programme ${ }^{29}$ » et « Compétences devant être acquises en fin de cycle ${ }^{30}$ ». Le

20. Ibid., p. 40.

21. Ibid.

22. Ibid., p. 41.

23. Ibid.

24. Ibid., p. 51.

25. Ibid., p. 59.

26. Ibid., p. 95.

27. Ibid., p. 89.

28. Ibid.

29. Ibid., p. 90.

30. Ibid., p. 93. Le terme « compétences » peut prêter à discussion, car il s'agit plus souvent de comportements à acquérir que de compétences. 
« programme » de cet apprentissage fondamental dénommé «Vivre ensemble » distingue trois points : "Dépasser l'horizon de l'école », "Continuer à construire sa personnalité au sein de la communauté scolaire », « Se construire comme sujet et comprendre sa place dans le groupe ». Dans le premier, « Dépasser l'horizon de l'école ${ }^{31} »$, figure notamment le texte suivant, qui se termine sur « l'appartenance à la communauté nationale » et sur l'importance de l'hymne national :

"L'école maternelle a déjà offert de nombreuses occasions de sortir de l'école et de s'ouvrir au monde. À l'école élémentaire, la multiplicité des domaines de connaissances abordés, de la première éducation littéraire ou artistique à la découverte du monde, permet d'amplifier cette ouverture et de la structurer.

« La rue, le quartier, la commune sont des transitions normales avec des espaces plus lointains. L'élève y découvre d'autres acteurs de la société qui jouent un rôle important dans sa vie quotidienne : agents de circulation, chauffeurs de bus, bibliothécaires, éducateurs sportifs, médiateurs culturels... Hommes et femmes occupent aujourd'hui également ces fonctions, ce qui n'a pas toujours été le cas dans le passé. Ils méritent respect et obéissance. Partout, des règles sont nécessaires. L'élève ne doit pas y voir des contraintes mais, au contraire, un moyen d'assurer la liberté, le bien-être et la sauvegarde de tous.

« Une attention particulière doit être apportée aux premières règles de sécurité routière, non pas sous forme de connaissances abstraites, mais à partir de situations quotidiennes vécues par les enfants à la sortie de l'école ou pendant les trajets scolaires. Leur aptitude croissante à lire, écrire et compter permet de lier cette éducation aux apprentissages fondamentaux.

«Le respect de l'environnement et du cadre de vie ne se limite pas à la classe et à l'école, mais s'étend à ces espaces publics qui sont le bien commun de tous. Au-delà, l'élève prend progressivement conscience de son appartenance à une communauté nationale à partir de l'écoute de quelques récits historiques et littéraires lus par le maître et en découvrant l'inscription de la France dans un espace géographique. L'enseignant lui explique la signification des grands symboles de la France et de la République : l'hymne national, le drapeau, quelques monuments ${ }^{32} \ldots \gg$

Dans le préambule, l'emploi de l'adjectif « national » pour qualifier la langue n'avait pas nécessairement de dimension identitaire; désormais, le doute n'est plus possible. Prendre " conscience de son appartenance à une communauté nationale à partir de l'écoute de quelques récits historiques et littéraires » 
ne peut se réduire au seul fait d'habiter ensemble sur le même territoire, dans le même pays. Cette appartenance signifie clairement l'héritage commun d'une histoire et d'une littérature, qui s'applique certes à un territoire qui en est marqué. Et tout cela se ramène à des symboles, parmi lesquels l'hymne national, qui donnent du sens à la nation. Cet hymne national fait par ailleurs l'objet du troisième alinéa de l'article 2 de la Constitution.

Selon la logique éditoriale de ce livre sur les programmes de l'école primaire, un encart de synthèse énonce à la fin du point II, intitulé «Vivre ensemble », les « compétences devant être acquises en fin de cycle ${ }^{33}$ » sur ce thème. Huit compétences sont précisées :

« Être capable de :

«- commencer à se sentir responsable ;

«- prendre part à un débat sur la vie de la classe ;

«- respecter les adultes et leur obéir dans l'exercice normal de leurs diverses fonctions.

«Avoir compris et retenu :

«- que les règles acceptées permettent la liberté de chacun, en particulier à partir de quelques exemples pris dans les règles de vie ;

«- quelques principes d'hygiène personnelle et collective et leur justification ;

«- quelques règles simples de sécurité routière ;

«- quelques règles à appliquer en situation de danger (se protéger, porter secours en alertant, en choisissant les comportements à suivre);

«- les principaux symboles de la nation et de la République ${ }^{34}$. »

La formulation de la huitième compétence devant être acquise confirme bien la dimension identitaire de la nation française, qui est certes un territoire, un État, plus précisément une République, mais aussi une nation, ce que l'enfant doit savoir au plus tard dès l'âge de neuf ans, avant de mieux encore assimiler cette question dans les années suivantes de l'école primaire.

Dans le cycle des approfondissements de l'école élémentaire, comprendre une hiérarchie d'appartenance

Dans le « cycle des approfondissements ${ }^{35}$ », deux domaines sont désignés comme « transversaux ${ }^{36}$ » : la «maîtrise du langage et de la langue française ${ }^{37}$ »

33. Ibid., p. 93.

34. Ibid.

35. Ibid., p. 147.

36. Ibid., p. 157.

37. Ibid., p. 159. 
et $l^{\prime}$ « éducation civique ${ }^{38}$ ». Le « programme » de cette dernière comprend quatre rubriques :

- « Participer pleinement à la vie de son école»;

- « Être citoyen dans sa commune »;

- « S'intégrer à l'Europe, découvrir la francophonie, s'ouvrir au monde » ;

- « Être citoyen en France ${ }^{39} »$.

Dans cette quatrième rubrique, il est précisé : « À travers les leçons d'histoire, l'élève comprend ce que signifie appartenir à une nation démocratique. La Déclaration des droits de l'homme et du citoyen ${ }^{40}$ est l'occasion d'aborder les articles qui concernent les diverses expressions de la liberté ${ }^{41}$. »

Cette formulation vise à transmettre une idée nouvelle : la France n'est pas seulement une nation, mais plus encore une nation démocratique fondée sur la liberté. Elle est d'autant moins une nation comme les autres qu'elle est la mère de la Déclaration des droits de l'homme et du citoyen.

Plus loin, à la fin de la présentation de ce domaine transversal qu'est l'éducation civique, sont précisées les « compétences devant être acquises en fin de cycle en éducation civique ${ }^{42} »$. Parmi les douze compétences énoncées, on peut lire :

«Avoir compris et retenu :

$«-[\ldots]$ ce qu'est un État républicain ;

«- ce que signifient l'appartenance à une nation, la solidarité européenne et l'ouverture au monde ${ }^{43}$.»

L'article « une » placé devant le mot « nation » pourrait signifier que tout individu de cette terre appartient à une nation, mais la suite de la phrase laisse entendre implicitement qu'il s'agit bien de la nation française, de l'appartenance à laquelle l'élève doit comprendre le sens, appartenance non exclusive de la « solidarité européenne » et de "l'ouverture au monde ». Cette phrase peut aussi être interprétée comme voulant indiquer une hiérarchie d'appartenance selon laquelle prime la nation par rapport à l'Europe et au monde. Autrement dit, l'élève doit se sentir d'abord français, et non d'abord européen ou citoyen du monde.

Toujours dans ce cycle des approfondissements figure un programme $d^{\prime}$ ' histoire ${ }^{44}$ » et un autre de « géographie ${ }^{45} »$ qui offrent chacun l'occasion d'utiliser le mot «nation » ou l'adjectif « national».

38. Ibid., p. 171.

39. Ibid., p. 173.

40. En italique dans le texte.

41. Ibid., p. 173.

42. Ibid., p. 175.

43. Ibid.

44. Ibid., p. 203.

45. Ibid., p. 211. 
L'histoire n'a pas seulement pour objet l'évocation du passé des hommes, des groupes dirigeants, des populations qui façonnent l'histoire ou du territoire qui connaît les soubresauts du temps ; elle se doit de traiter du «passé national ${ }^{46} »$, qui s'inscrit dans une temporalité bien précise. Cette dernière ne correspond certes pas aux deux plus anciennes périodes historiques qui sont à enseigner, « la préhistoire » et «l'Antiquité », mais au « Moyen Âge (476-1492)». La datation est évidemment l'occasion de souligner l'ancienneté de la nation française et tout autant de sa capitale : « À partir du IV siècle, des peuples venus de l'est, notamment les Francs et les Wisigoths, s'installent dans l'Empire romain d'Occident, qui s'effondre définitivement vers la fin du v'e siècle. Sur ces ruines s'établissent des royaumes fondés par des peuples germaniques. Cette période est décisive dans notre passé national, avec le nom même de notre pays, l'émergence de sa capitale et, en même temps, des grandes identités régionales ${ }^{47}$. » En revanche, parmi les « compétences devant être acquises en fin de cycle » pour l'histoire, le mot «nation » n'apparaît pas, ce qui peut être interprété comme une lacune relativement au souci d'enseigner la nation, ou tout simplement par le fait que l'acquis a déjà été consigné dans le cycle des apprentissages fondamentaux.

Quant à la géographie, elle semble moins participer à l'enseignement de la nation, car elle apparaît déconnectée des réalités humaines, sociales et culturelles. En effet, la géographie de l'école primaire est essentiellement descriptive et se contente simplement de lire les paysages, de souligner combien l'homme transforme la morphologie de l'espace. Aucune place ne semble faite à des notions qui, selon les intitulés des différentes branches de la géographie, se rattacheraient à la géographie historique, à la géographie culturelle ou à la géographie politique.

« À cette étape de la scolarité, la géographie commence par une sensibilisation à la diversité des espaces, pour arriver à une lecture des paysages et des représentations cartographiques qui en rendent compte. Le parcours va du cadre le plus large, le monde, pour recentrer l'attention sur la France, en passant par l'Europe. Descriptions et comparaisons permettent de mettre en lumière quelques-unes des manières dont l'homme transforme l'espace qu'il habite. La géographie facilite un rapport concret et actif à l'espace et à ses représentations ${ }^{48}$. » Même si elle permet de souligner combien les territoires, singulièrement les territoires français, sont riches de leur diversité, la discipline géographique, selon les programmes de 2004, n'est guère utilisée à l'école primaire à des fins d'enseignement de la nation comme elle l'était sous la III ${ }^{\mathrm{e}}$ République. 


\section{La nation au premier rang de l'un des vocabulaires à retenir}

Enfin, dans ce livre sur l'école élémentaire, le ministère de l'Éducation nationale, selon sa pratique habituelle, propose des « documents d'application et d'accompagnement ${ }^{49}$ ». Ceux-ci précisent notamment les " événements, dates, personnages, vocabulaire et documents correspondant au programme d'histoire du cycle $3^{50}$ ». Pour le « XIX siècle $^{51}$ », dans le « vocabulaire à retenir », les mots sont placés dans l'ordre suivant : " nation, suffrage universel, colonies, bourgeois, industrialisation, émigration, exode rural, ouvriers, syndicats, grève, socialisme, impressionnisme, métropolitain, électricité ${ }^{52} \gg$. Cette liste ne respecte pas l'ordre alphabétique. Les mots seraient-ils classés selon un ordre historique ou selon un ordre d'importance dans la connaissance que l'élève doit en avoir ? Chercher une réponse à cette question laisse dubitatif. Pourquoi, par exemple, le mot «bourgeois » aurait-il plus d'importance que le mot « industrialisation»? Le lecteur lambda finit par conclure que cette liste s'inscrit dans un certain désordre, mais une certitude demeure : la première place accordée au mot «nation » dans la liste. Cependant, puisque nous sommes dans le vocabulaire à retenir pour l'histoire du $\mathrm{XIX}^{\mathrm{e}}$ siècle, il ne s'agit guère là de la nation française, mais de la création, à cette époque, de nations par le remplacement, sur plusieurs terres européennes, de «pouvoirs monarchiques, souvent de droit divin, par des pouvoirs fondés sur la volonté générale et la souveraineté du peuple ${ }^{53} »$.

Finalement, même s'il a connu des évolutions, l'enseignement de la nation demeure clairement, pour les élèves de six à onze ans, un objectif ambitieux puisqu'il s'agit d'enseigner différents sens du mot nation, notamment son sens politico-culturel, c'est-à-dire celui d'une collectivité humaine unie par le sentiment de sa continuité, d'un passé partagé, d'un avenir commun, d'un héritage identitaire à transmettre et de valeurs à respecter.

Puisque la nation est enseignée dans le primaire, sa place dans le secondaire n'est pas assignée de façon aussi directive, mais elle est évidemment présente tant au collège qu'au lycée à travers les enseignements d'histoire, de géographie ou d'instruction civique.

49. Ibid., p. 279.

50. Ibid., p. 332.

51. Ibid., p. 333.

52. Ibid., p. 335.

53. Cf. Pierre Nora, Guide républicain, Paris, SCÉREN/Delagrave, 2004. 


\section{Deux propos de parlementaires sur l'enseignement de l'hymne national de la France}

«L'hymne national fait partie de notre histoire. »

« La Marseillaise appartient à notre patrimoine. Il est extrêmement important et légitime que tous les enfants de France connaissent l'hymne national, qui fait partie de notre histoire. »

Jack Lang, député PS, ancien ministre de l'Éducation nationale, France-Soir, 22 février 2005.

«La Marseillaise à l'école ? Pour un moment de ralliement qui marque l'adhésion à notre société. »

«Ce qu'exige la loi, c'est non pas d'apprendre La Marseillaise par cœur, mais de l'enseigner. L'hymne national doit être appris comme on le ferait avec un poème de Prévert, par exemple : on étudie les mots, l'histoire du poète, l'époque. On tourne autour du texte. Il ne s'agit pas, chaque matin, de mettre les enfants en rang par deux dans la cour de l'école pour chanter La Marseillaise. Mais l'école a un rôle primordial dans la transmission des valeurs de la République. Il est donc normal d'enseigner l'hymne national qui les exalte, de redire quelles sont ces valeurs, c'est-à-dire la liberté individuelle et le respect de l'autre. »

Jérôme Rivière, député UMP des Alpes-Maritimes, L'Express, 28 février 2005.

\section{Questionnements}

La lecture de l'ensemble des programmes éducatifs conduit à différents questionnements. La réponse apportée au premier, portant sur l'appartenance de la France à l'Union européenne, peut s'examiner à travers les programmes, sans que cette réponse, néanmoins, apparaisse totalement satisfaisante. En revanche, les autres questionnements sont plus généraux et se présentent comme de véritables défis pour l'avenir de l'enseignement de la nation.

\section{Faire place à l'Europe}

La construction européenne introduit une nouvelle dimension identitaire audelà, bien évidemment, du référent national. L'Union européenne ne remplace pas les différentes nationalités qui la composent ; elle demande en revanche que soit mené un travail d'appropriation du sentiment d'appartenance à ce nouveau 
repère territorial : «On ne naît pas européen, on le devient », déclarait Václav Havel en $1995^{54}$.

Dans ce contexte de recomposition spatiale à une autre échelle, l'enseignement de la géographie devrait proposer une réflexion sur l'appartenance identitaire et participer à sa conscientisation. Mais l'Europe n'a pas d'identité territoriale clairement définie : à quelle Europe se réfère-t-on ? Comme le démontrent ses élargissements successifs, l'espace européen n'est pas reconnu par les dirigeants comme un territoire fini. Cette identité géographique en mouvement rend difficile l'élaboration d'un sentiment d'appartenance. Les incertitudes quant à l'idéal politique, économique et social à atteindre au terme de la construction européenne renforcent les difficultés à construire une représentation commune de l'Europe.

\section{Au collège, une identité nationale en rien incompatible avec l'identité européenne}

Les questions soulevées par l'inclusion de l'Europe dans les programmes scolaires conduisent aussi à réfléchir à l'enseignement de la nation. Voici par exemple ce qu'en pense l'inspection générale de l'Éducation nationale : «En classe de troisième des collèges, l'Europe est inscrite au programme à travers les conflits et les bouleversements géopolitiques qui les suivent : étude des nouvelles cartes de l'Europe, en 1914, 1939, 1942 et 1945. En géographie, on aborde la question spécifique de l'Union européenne dont on étudie la construction et que l'on présente, aux côtés des États-Unis et du Japon, comme une des trois grandes puissances économiques de la planète. Le traité de Rome (1957), celui de Maastricht (1992) et la carte des États membres avec leurs capitales apparaissent dans la liste des repères à mémoriser pour le diplôme national du brevet. »

«Les institutions européennes, mais aussi et surtout le concept de citoyenneté européenne tel qu'il se dégage du traité de Maastricht, sont inscrits au programme d'éducation civique de troisième. Il s'agit bien ici de faire connaître les valeurs universelles et humanistes nées en Europe, la liberté comme "patrimoine commun d'une Europe fragmentée", le respect des droits de l'homme. Il s'agit aussi de montrer que, dans le cadre d'une diversité assumée, l'identité nationale n'est pas incompatible avec une identité européenne qui est à la fois héritage et avenir à construire ${ }^{55}$. »

54. Cf. Hélène Béchet, La Dimension européenne de l'enseignement de la géographie dans le système scolaire secondaire : l'exemple de la France et de l'Espagne, Université Paris IVSorbonne, mémoire de DEA de géographie culturelle, historique et politique, juin 2004.

55. Inspection générale de l'Éducation nationale, Groupe histoire et géographie, rapport de discipline ou de spécialité 1999-2000, «L'Europe dans l'enseignement de l'histoire, de la géographie et de l'éducation civique », sept. 2000. 
Cependant, la mise en œuvre des contenus européens des programmes dans les collèges n'est pas aisée, comme le précise l'inspection générale : «En éducation civique, les professeurs et leurs élèves peinent à se percevoir comme citoyens - ou futurs citoyens - européens tant la citoyenneté leur apparaît indissociable de l'appartenance à un État-nation. La Convention européenne des droits de l'homme et des libertés fondamentales, le traité de Maastricht et quelques cartes sont les éléments de référence les plus mentionnés des leçons sur l'Europe, mais rares sont les leçons échappant à la simple description des institutions et des mécanismes de l'Union européenne. »

«Encore faudrait-il que le concept de "citoyenneté européenne" ne demeure pas vague et indéterminé et que sa définition et son acquisition figurent plus nettement encore dans les finalités avouées de nos enseignements d'éducation civique. »

"À l'évidence "le souffle européen" est un peu court et l'esprit européen encore bien discret. L'Europe demeure pour la plupart des enseignants une question difficile à problématiser dont on ne discerne pas les objectifs. N'est-ce pas finalement le reflet au niveau des enseignants des hésitations d'une opinion publique pour le moins partagée ? L'enseignement de l'Europe reste donc un objet en débat et en projet parce que c'est un objet politique inachevé. Il nous faut encore beaucoup travailler pour en achever l'invention. Notons, dans ce contexte, que semblent datées les virulentes accusations d'européocentrisme des programmes et des manuels formulées dans les années soixante-dix et quatrevingt. »

« À l'issue de cette observation, deux questions apparaissent auxquelles les professeurs d'histoire et de géographie, notamment, sont confrontés. La première est essentiellement politique : faudrait-il, dans une démarche volontariste et dans le dessein de susciter un sentiment d'appartenance européenne, articuler les enseignements d'histoire, de géographie et d'éducation civique comme le fit consciemment la III ${ }^{e}$ République pour renforcer le sentiment national ${ }^{56}$ ?»

\section{La géographie au lycée : entre la primauté donnée au référent national ou à un espace réticulaire}

Concernant la géographie au lycée, nous observons deux approches du territoire : la première laisse prédominer le sentiment national, la seconde l'efface pratiquement.

Prenons l'exemple de la classe de première du lycée. Les questions majeures qui se sont posées au groupe d'experts lors de l'élaboration du programme 
furent : Quelle Europe étudier ? Où s'arrêtent ses limites ? Comment l'appréhender ? Le choix du programme aurait pu s'orienter soit vers la présentation d'une Europe des États, soit vers une étude globale de l'espace européen. La réflexion du groupe a finalement abouti à une combinaison des deux.

La première partie du programme de première, «L'Europe des États », confirme la prééminence du cadre d'étude national. « Aujourd'hui, les frontières des États constituent le maillage essentiel de l'organisation de l'espace politique européen ${ }^{57}$. » L'Europe est présentée non pas comme un territoire, mais comme un espace, choix délibéré qui prouve la difficulté des concepteurs à dépasser la référence territoriale nationale.

Quant à la construction européenne, elle est présentée comme un «système institutionnel d'alliance et de coopération entre les États-nations souverains et démocratiques ${ }^{58} \gg$. L'historique de cette construction s'appuie sur la chronologie des adhésions successives des États et insiste particulièrement sur le traité de Maastricht (février 1992).

Dans l'un des manuels cités à titre d'exemple, pour montrer les limites de la portée de ce traité, c'est avant tout la position française qui est exposée : «Le référendum français n'obtient qu'une faible victoire du oui. L'Europe n'est pas ancrée dans la vie quotidienne des Français, mais considérée comme l'œuvre de technocrates bruxellois ${ }^{59}$. » Le choix fait par les auteurs de présenter une adhésion peu motivée des Français à l'Union européenne révèle la prééminence d'un fort sentiment national, ce que confirme la formulation suivante : «Pour l'État français, l'Europe a pour fonction de garantir et d'amplifier la puissance nationale ${ }^{60}$. $\gg$ La préservation des intérêts nationaux est donc mise en avant : les choix nationaux doivent prévaloir sur les décisions communautaires. L'Europe politique n'est «ni une nation ni un État : elle institue des lois qui s'imposent par les traités aux lois nationales ${ }^{61} \gg$. Le déficit de légitimité démocratique est également souligné, ainsi que la crainte de voir disparaître les spécificités nationales culturelles, le savoir-faire et la conception du service public.

L'approche privilégiée par les auteurs porte sur la difficulté d'éveiller une conscience politique et citoyenne européenne : le référent national demeure pour les Français le cadre de référence essentiel et l'Union européenne est présentée comme une entité qui est loin d'être intégrée dans le quotidien et dans le cœur des citoyens.

57. Bulletin officiel du ministère de l'Éducation nationale, hors-série $\mathrm{n}^{\circ} 73$, oct. 2002.

58. Ibid.

59. Annette Ciattoni (sous la direction de), Manuel de géographie de Première, Paris, Hatier, 2003.

60. Ibid.

61. Ibid. 
Dans la suite du programme, l'étude comparative approfondie de deux États européens permet aux élèves de comprendre d'autres fonctionnements politiques et territoriaux que ceux de la France et de prendre conscience que le modèle français n'est ni unique ni prépondérant en Europe.

Ainsi la première partie du programme de première s'appuie-t-elle sur une approche nationale de « l'espace européen », l'État-nation demeurant le cadre de référence de ce premier quart du programme.

La démarche privilégiée dans la deuxième partie, "Réseaux et flux en France et en Europe », s'avère radicalement différente : loin de s'arrêter aux frontières nationales, c'est sur l'espace « déterritorialisé » que portent les problématiques et les thèmes proposés.

«L'objectif de la deuxième partie est de faire comprendre qu'au-delà du cadre des États, l'espace européen est également organisé selon la logique de fonctionnement en réseau qui relève du libre jeu des acteurs économiques et sociaux dans un espace ouvert. L'effacement progressif des frontières renforce les effets de ces logiques à l'échelle du continent ${ }^{62}$. » Cette partie souligne donc l'ouverture du marché européen, le rôle du libre jeu des acteurs économiques et la perte de l'importance de la notion de frontière.

La deuxième partie s'appuie davantage sur une approche conceptuelle, avec un vocabulaire spécifique à intégrer : flux, carrefour, réseau, axes, semis urbain, maillage, polarisation, métropolisation, mondialisation...

Les réseaux urbains et les métropoles sont l'objet d'une première étude. C'est avant tout le caractère transfrontalier et international qui est mis en avant. « L'objectif européen d'un espace de circulation intégré impose de dépasser les logiques nationales, parfois contradictoires, d'organisation du territoire ${ }^{63}$. » La constitution d'un réseau « paneuropéen ${ }^{64}$ » de transports et de télécommunications est présentée comme une nécessité d'adaptation à la logique de marché, ce qui implique « la dérégulation des transports aériens, des télécommunications, et la privatisation d'entreprises publiques ${ }^{65} »$.

Le second thème étudié porte sur « la mobilité des hommes ». Les flux internationaux de population, induits par des facteurs économiques et politiques, entrainent un brassage de population qui tend à modifier les identités nationales.

Ainsi, l'espace européen, au regard de cette deuxième partie, se définit non plus par rapport aux États qui le composent, mais par son intégration dans un

62. Bulletin officiel du ministère de l'Éducation nationale, op. cit.

63. Ibid.

64. Roger Brunet, Daniel Pierre-Élien (sous la direction de), Manuel de géographie Première, Paris, Bréal, 2003.

65. Ibid. 
marché économique mondial : la modernité est dépassée par une vision « déterritorialisée » de la construction européenne.

Concernant l'enseignement de l'histoire au lycée, des initiatives ont été prises pour favoriser une « histoire européenne », et donc intégrer l'enseignement de la nation dans le champ européen. Ainsi Dominique Strauss-Kahn a-til remis le 18 mai 2004 au président de la Commission européenne, Romano Prodi, un rapport intitulé : «Construire l'Europe politique ». L'une des propositions porte sur l'enseignement de «l'histoire européenne », mais la possibilité d'organiser également un cours de « géographie européenne ${ }^{66} »$ n'est nullement mentionnée.

En outre, depuis les années 1970, le Conseil de l'Europe entreprend de réconcilier les histoires nationales trop conflictuelles : entre l'Allemagne et ses voisins, puis, plus récemment, dans le Caucase et les Balkans. Depuis les années 1980, un manuel d'histoire commun franco-allemand est en chantier. Le projet réapparaît régulièrement pour, chaque fois, se heurter aux mêmes obstacles : « le rôle différent des manuels, recueil de documents en Allemagne, mais charnière des cours de l'éducation nationale en France ; les programmes nationaux en France, mais régionaux (par Land) outre-Rhin, et la difficulté de s'entendre sur le contenu du manuel », selon Michel Cullin, secrétaire général adjoint de l'Office franco-allemand pour la jeunesse. Malgré ces difficultés, Jacques Chirac et Gerhard Schröder ont annoncé, lors du sommet de Poitiers d'octobre 2003, l'élaboration d'un manuel d'histoire. Une commission d'historiens s'est réunie et a arrêté le plan de l'ouvrage. Mais plusieurs décisions restent en suspens : le choix d'un éditeur prêt à prendre le risque financier d'un ouvrage à la diffusion incertaine et le choix entre deux éditions ou une seule, bilingue.

Plus récemment, le 24 novembre 2004, les personnalités réunies à Londres sous l'égide du Conseil franco-britannique ont approuvé à l'unanimité une proposition recommandant la réalisation d'un manuel commun d'histoire franco-britannique, à l'instar du manuel commun d'histoire franco-allemande précité. Elles espèrent une décision d'un sommet franco-britannique afin de faire travailler un groupe d'experts, déjà constitué sous la responsabilité du professeur Robert Franck, de l'université Paris I - Panthéon-Sorbonne, pour rédiger un ouvrage scolaire en définissant le public cible le mieux adapté (primaire, collège ou lycée) et les solutions techniques requises pour une diffusion crédible.

Même si un enseignement de géographie européenne commun s'avère nécessaire, il est important de conserver une spécificité nationale aux

66. Cf. Nicolas Chevassus, « La vieille Europe, continent sans histoire », Le Monde de l'Éducation, $\mathrm{n}^{\circ} 325$, mai 2004. 
programmes et à la pédagogie de l'enseignement de la géographie. Définir un contenu commun et une approche similaire de la géographie européenne porterait atteinte aux spécificités nationales et tendrait à « appauvrir » la diversité culturelle qui fait la richesse de l'Union européenne.

S'il est vrai que la mentalité française repose sur le bon sens et sur le culte de la raison, la population devrait se laisser convaincre par la force des arguments soulignant la compatibilité des cultures française et européenne. S'il est vrai que la mentalité nationale comporte un élan vers l'universalisme et vers la transmission d'un message, les Français pourraient être tentés de contribuer activement au façonnement d'une Europe correspondant à leurs aspirations. La tâche se révélerait d'autant plus aisée qu'en de nombreux domaines, droits de l'homme, liberté, démocratie, séparation des pouvoirs, les points de vue sont proches, voire identiques.

Le rapprochement entre la France et l'Europe apparaît encore plus logique et propre à flatter la fierté hexagonale si l'on considère que, sur le plan symbolique, notre pays, situé au cœur du vieux continent, juxtapose en un raccourci étonnant tous les caractères naturels, relief, climat, hydrologie, de cette région du monde : la France est un modèle de la géographie européenne. En outre, c'est là que se sont mêlés des peuples très divers : la France est un résumé ethnique de l'Europe.

La vocation européenne de la France possède donc tous les caractères de l'évidence. Il semble qu'il faille en convaincre nombre de Français. Aussi la tâche prioritaire est-elle de définir une pédagogie de l'identité européenne.

L'enseignement de la nation se trouve donc inévitablement à repenser compte tenu de la construction européenne, même si cette nécessaire évolution ne préoccupe pas ceux des partisans de l'Europe qui soulignent le lien indissoluble entre la spécificité française et la communauté culturelle que forment les peuples du vieux continent ${ }^{67}$. Mais d'autres questionnements apparaissent, comme celui de la crise de l'identité nationale.

\section{La crise de l'identité française et l'enseignement de la nation}

Certains des maux qui traversent la société française peuvent comporter des risques de désagrégation du sens de la nation. En effet, enseigner la nation suppose logiquement d'avoir une vision claire de son identité. Or l'identité traditionnelle de la France se trouve mise en cause pour diverses raisons. Les épreuves de la décolonisation, l'affaiblissement de l'influence internationale de la France, le ralentissement de sa croissance économique, le chômage, les frac-

67. Cf. Gérard-François Dumont, L'Identité de l'Europe, Nice, CRDP, 1997. 
tures sociales, la perte d'influence des structures traditionnelles d'encadrement (école, partis, syndicats, Église), la présence d'une population immigrée ou issue de l'immigration mal connue, la construction d'une Europe qui n'est pas toujours acceptée, la mondialisation de la culture, l'anémie des idéologies qui, jadis, avaient mobilisé d'importants effectifs, tous ces facteurs engendrent, selon les cas, pessimisme, désarroi, angoisse face à l'avenir, repli sur la sphère du privé. Des expressions familières comme «bof génération » ou « soft génération » sont apparues dans les années 1980 et 1990. Publié en 1983, L’ère du vide, de Gilles Lipovetsky, dépeint une population française individualiste et dépourvue de projet collectif. En 1993, Alain Duhamel analyse Les Peurs françaises, autre titre significatif. L'idée se répand que le pays vieillit, c'est une France ridée, comme l'annonce dès 1979 Gérard-François Dumont ${ }^{68}$, en collaboration avec Alfred Sauvy et Pierre Chaunu. Il découle de tout cela une certaine crise de la conscience nationale qui amène les citoyens à réfléchir à l'identité. Certains y voient une valeur-refuge ; d'autres la jugent trop affaiblie pour qu'elle puisse offrir une sauvegarde aux Français désorientés ; quelquesuns en profitent pour contester tout ou partie des principes traditionnels.

Enfin, les régionalistes contestent la toute-puissance de l'État-nation et dénoncent l'oppression dont celui-ci se serait rendu coupable à leurs yeux. Ils lui reprochent d'avoir imposé une langue, une culture, des mythes qui ont étouffé les particularités régionales. Aussi veulent-ils profiter de la crise pour restaurer les valeurs et les usages jadis malmenés.

Dans ce contexte, on saisit toute la difficulté à enseigner la nation, mission rendue sans doute plus délicate auprès de populations issues de l'immigration.

\section{Immigration et enseignement de la nation}

Certes, dès le milieu du XIX ${ }^{\mathrm{e}}$ siècle, l'immigration prend une importance considérable en France ${ }^{69}$. Depuis, pour pallier une natalité insuffisante et les pertes humaines dues aux conflits mondiaux, pour disposer d'une main-d'œuvre nécessaire au relèvement des ruines accumulées par les guerres, pour soutenir l'expansion économique, pour rester fidèles à la réputation qu'a le pays de havre pour les populations persécutées, les gouvernements successifs ont favorisé l'entrée des étrangers et de leurs familles. Aux Européens, encore majoritaires dans la population étrangère résidant en France selon le recensement de 1936, succèdent, depuis la Seconde Guerre mondiale, des contingents de plus en plus

68. Cf. Gérard-François Dumont et coll., La France ridée, Paris, Livre de poche, 1979, et Paris, Hachette (Pluriel), 1986, pour la seconde édition.

69. Cf. Gérard-François Dumont, La Population de la France, des régions et des DОм-TOM, Paris, Ellipses, 2000. 
nombreux d'Africains et d'Asiatiques. Au recensement de 1931, les étrangers sont 3 millions. Au recensement de 1990, ils sont 3,58 millions, soit 6,4\% de la population totale. Au recensement de 1999, on compte 3,263 millions d'étrangers et 4,309 millions d'immigrés ${ }^{70}$. Dans les années 2000, chaque année, quelque 200000 immigrés entrent en France ${ }^{71}$, tandis que 100000 autres deviennent français par divers processus.

D'où la question : comment enseigner la nation lorsque beaucoup d'élèves, issus de mondes étrangers, éprouvent des difficultés à se reconnaître dans l'histoire de France et à s'identifier à nos ancêtres de l'Hexagone ?

Dans le même temps, sous l'effet des évolutions idéologiques, l'enseignement de la nation doit en outre faire face à deux idéologies opposées mais objectivement alliées : le communautarisme, favorisé par l'importance des flux migratoires, et l'individualisme, qui omet le sens du bien commun.

\section{L'individualisme ou le communautarisme versus le sens collectif}

Le communautarisme, qui, dans une société davantage pluriethnique, peut l'emporter sur le désir de s'intégrer, semble offrir une alternative à la morale républicaine. Mais, jouant sur les réflexes identitaires, religieux ou ethniques, il détricote la nation, tout particulièrement lorsqu'il se diffuse par l'endoctrinement de quelques jeunes issus de l'immigration par des prêcheurs fondamentalistes. Il existe aussi un communautarisme idéologique, ce que deux militants de gauche appellent «l'horreur démagogique ${ }^{72} »$. Cela concerne des groupes balayant l'intérêt général qui, dans la sphère publique, devrait prévaloir sur les particularités. De tels groupes se consacrent alors exclusivement à la défense des minorités susceptibles de l'emporter sur le fait majoritaire ou à l'organisation de moyens de pression, et utilisent souvent le langage de la victime pour construire l'action politique, éprouvant la légitimité de la représentation nationale.

Ensuite se pose la question de l'individualisme. La démocratie, par son essence même, est fragile parce que, justement, elle implique l'adhésion de tous et ne saurait se satisfaire du désintérêt d'individus repliés sur leur sphère privée. Elle est fragile parce qu'elle est exigeante ; elle demande à l'homme, qui est d'abord un individu, qu'il soit aussi un citoyen susceptible de dépasser ses préoccupations personnelles pour penser l'intérêt de la collectivité. Parce que la

70. Selon la définition du Haut Conseil à l'intégration. Cf. Gérard-François Dumont, Gabriel Wackermann, Géographie de la France, Paris, Ellipses, 2002.

71. Cf. Xavier Thierry, «Évolution de l'immigration en France et éléments de comparaison avec le Royaume-Uni », Population, vol. 59, n 5, 2004.

72. Cf. Guy Dhoquois, Régine Dhoquois, Le militant contradictoire, Paris, L'Harmattan, 2005. 
citoyenneté ne peut qu'être collective, on ne peut pas être citoyen tout seul et l'individualisme ne peut faire vivre la cité, qui repose d'abord sur le sens du bien commun.

Or, dans un Hexagone qui subit des chocs comme la diffusion subtile d'idéologies déstabilisant la société, le chômage ou la précarité, ce dédoublement devient difficile et l'individu tend à l'emporter sur le citoyen. D'autant que le discours dominant véhiculé par les médias met en avant le temps libre, le cocooning et l'hédonisme. Une idéologie narcissique est souvent célébrée par la publicité, poussant à une uniformisation des modes de vie ${ }^{73}$ et de pensée caractérisée par la vulgarité, le goût de la violence, de la provocation. Sont souvent privilégiés le souci de paraître plutôt que d'être, l'apologie de la combine, où il vaut mieux être " cool » que consciencieux. Dans ce contexte, quelle place reste-t-il pour la vertu et l'engagement citoyen fidèle au sentiment national ?

\section{Les valeurs inversées de la télé-réalité}

On peut par exemple s'interroger sur le contexte médiatique, dont les émissions de télé-réalité ${ }^{74}$ véhiculent des valeurs inverses de celles qu'appelle la nation. La vie en collectivité, pour qu'y règne la concorde sociale, suppose la politesse et la pudeur, au sens le plus noble de ces termes. Or une émission comme La Ferme ne présente que l'inverse et valorise des chamailleries incessantes. Même si certains peuvent penser que Star Academy n'est qu'un radiocrochet modernisé, l'émission se situe dans une perspective beaucoup moins humaine, tout étant organisé sur des principes selon lesquels il s'agit d'assurer la promotion de produits pour qu'ils deviennent objets d'une grande consommation. Si nous regardons maintenant du côté de L'île de la tentation, émission qui vise à défaire des couples, les valeurs proposées sont à l'inverse de l'esprit qui doit prévaloir dans la nation. Le cadre de l'émission propose des lieux de villégiature sans identité culturelle, donc des territoires déshumanisés, pour parvenir à faire renoncer à cette sorte de patriotisme qu'est le patriotisme amoureux. Enfin, Bachelor, l'émission où un homme choisit sa femme dans un troupeau de femelles, propose un modèle d'éducation amoureuse où le respect de l'autre laisse la place à un machisme animal.

73. Contraire à la maxime de La Bruyère : «Il faut faire comme tout le monde : maxime qui signifie presque toujours : il faut mal faire. »

74. Cf. Damien Le Guay, L'Empire de la télé-réalité, ou comment accroître le " temps du cerveau humain disponible », Paris, Presses de la Renaissance, 2005. 


\section{La globalisation ou l'américanisation}

Un autre questionnement provient d'une certaine mondialisation de la culture sur le modèle américain, de son américanisation. Dans un sens général, l'américanisation peut se définir comme un rapport à l'Amérique, en fait aux États-Unis considérés comme la concrétisation de ce que fut le rêve américain avant même la création des États-Unis. Dans un sens plus précis et plus opérationnel, l'américanisation se définit non seulement comme l'adoption de manières de vivre, de penser et d'agir conformes à celles des États-Unis par des peuples non américains, mais aussi comme la substitution de ces manières à des cultures spécifiques et à des identités qui contribuaient à la richesse humaine et culturelle du monde ${ }^{75}$.

Cette américanisation provient de processus encouragés à la fois par les émetteurs américains et par l'attitude des récepteurs. Les premiers - les émetteurs américains - sont imbus d'une croyance en leur rôle mondial pouvant aller jusqu'à une tentation hégémonique, comme l'attestent par exemple le rapport Kissinger (rédigé en 1974 et longtemps resté secret) ou, plus récemment, les rapports Wolfowitz (établi par le Pentagone) et Jeremiah (émanant d'un groupe d'experts), cités dans le New York Times en 1992. Dans ces derniers documents, on peut lire par exemple le souci des États-Unis d'empêcher «l'émergence d'un système de sécurité exclusivement européen qui pourrait déstabiliser l'OTAN ».

Les seconds - les récepteurs -, trop souvent oublieux des racines de leur identité et de leur culture, se font souvent les acteurs des aspects nocifs de leur propre américanisation du fait de nombre de décisions ou de non-décisions politiques et de comportements qui sont facilités, voire encouragés.

Un résumé de cette influence américaine peut être le suivant. Dès le lendemain de la Seconde Guerre mondiale, le jazz triomphe dans les caves de SaintGermain-des-Prés. Puis s'imposent les films, le «polar », la science-fiction venus des États-Unis. Bientôt arrivent des vêtements comme le « jean », la nourriture rapide ou "fast-food », les séries télévisées ou "soap opera », des musiques populaires, les grandes surfaces en bordure des villes. L'influence américaine s'exerce encore dans certaines sciences humaines comme la sociologie, la publicité, les études de marché ou «marketing », les méthodes de gestion ou « management ». L'importance des mots anglais dans le vocabulaire scientifique et technique, dans les activités du secteur tertiaire, traduit également le poids du Nouveau Monde.

75. Cf. Gérard-François Dumont, «L'américanisation de l'Europe », Revue française de géopolitique, $\mathrm{n}^{\circ}$ 1, 2003, p. 197-202, repris dans le $\mathrm{n}^{\circ}$ 3, éd. 2005. 
L'américanisation, incontestable sous certains aspects, s'effectue au $\mathrm{XXI}^{\mathrm{e}}$ siècle dans un contexte de globalisation, fruit de décisions politiques nationales, régionales et internationales, dont le rythme s'est trouvé facilité par l'essor de procédés favorisant les échanges entre les différents territoires de la planète, c'est-à-dire l'internationalisation.

La globalisation et l'internationalisation sont donc des processus déjà largement reconnus et, en conséquence, devenus des paramètres, politique pour le premier et techno-géographique pour le second. Dans la mesure où la puissance américaine, par son poids géopolitique et industriel, demeure dominante dans l'évolution de ces deux processus, certains aspects de l'américanisation de la France et de l'Europe sont sans doute inévitables, et même souhaitables. Sauf à refuser, sous prétexte qu'ils sont une invention américaine, le courrier électronique ou l'internet, qui représentent pour l'humanité un saut qualitatif tout aussi important que celui qu'a permis Gutenberg. Aussi appartient-il à la France et à l'Europe, aujourd'hui et demain, de dire oui aux mesures de globalisation lorsqu'elles sont favorables aux hommes et de dire non à celles qui sont contraires à ce que j'appelle la « culturo-diversité ${ }^{76}$ » et aux valeurs d'une nation française acteur de l'Europe, au risque de tomber dans le complexe de Procuste ; car le monde a besoin de cette culturo-diversité comme de la biodiversité. En effet, l'avenir de la France et des peuples d'Europe s'inscrit dans la capacité à s'enrichir et à enrichir un sentiment national et européen, c'est-à-dire à renouveler et à promouvoir les valeurs qui ont forgé leur identité.

C'est un défi pour l'éducation que de préserver et de promouvoir la richesse de l'identité française en évitant que celle-ci ne disparaisse sous les assauts de modes américaines dont les effets ne sont pas seulement de façade. L'éducation doit viser une parfaite maîtrise de la langue française et une intériorisation de la culture du pays natal, car ce sont là les meilleurs atouts pour l'équilibre des liens sociaux, pour être reconnu et apprécié, pour être capable d'un dialogue fructueux avec d'autres cultures. Toute communication entre deux cultures se révèle encore plus riche si, de chaque côté, on possède des fondements intellectuels solides. D'ailleurs, comment bien apprendre une langue étrangère si l'on maîtrise imparfaitement son propre idiome?

\section{Citoyenneté active ou passive?}

Un dernier questionnement provient du fait que la citoyenneté semble avoir progressivement perdu une partie de son contenu civique : on est passé d'une vision idéalisée de la souveraineté nationale à un système de garantie des droits

76. Cf. Gérard-François Dumont, «Unité du genre humain et diversité des civilisations », Panoramiques, $\mathrm{n}^{\circ}$ 68, $3^{\mathrm{e}}$ trim. 2004. 
de l'individu. Pourtant, les droits des citoyens français sont très clairement affirmés dans la Déclaration de 1789 et dans le préambule de la Constitution de 1946, confirmés par le préambule de la Constitution de 1958. Et ce ne sont en rien des droits formels, mais des droits réels auxquels la jurisprudence recourt périodiquement pour fonder ses décisions.

Or trop de citoyens semblent aujourd'hui participer à une certaine course aux droits, droits dont le nombre semble croissant. Ceux-ci sont peut-être individuellement légitimes, mais une nation peut-elle être la somme de droits individuels qui, d'ailleurs, se contredisent jusqu'à masquer l'essentiel ? Heureusement, cette course aux droits échoue parfois, comme en 1989, lorsque certains avaient imaginé remplacer la Déclaration des droits de l'homme et du citoyen de 1789 par une nouvelle déclaration des droits, alors que la valeur d'un tel texte tient à son caractère non relatif, à sa vertu pérenne.

La course aux droits individuels repose sur l'idée que ceux-ci ne peuvent résulter d'une règle collective, mais doivent faire l'objet d'une inscription législative spécifique. Elle provoque d'autres conséquences dommageables dans la mesure où la seconde revendication essentielle, qui devrait être attachée aux droits comme le revers d'une pièce de monnaie ne saurait être dissocié de son avers, la revendication des devoirs, semble omise.

On peut donc s'interroger sur la crise de la citoyenneté. Il existe d'un côté le civisme actif, qui sait mettre en parallèle droits et devoirs, et qui se trouve en recul. À l'opposé se développe une vraie « fausse » citoyenneté, une citoyenneté passive, qui consiste exclusivement à revendiquer ses droits.

Or, dans le dessein d'enrayer de telles évolutions, enseigner la nation consiste à rappeler que la citoyenneté passe par la mise en œuvre des vertus républicaines, c'est-à-dire des valeurs de la République et de la défense des libertés. Il s'agit à la fois d'apprendre la citoyenneté active par l'éducation civique et, plus généralement, par un bon fonctionnement du système éducatif, mais aussi de savoir que la citoyenneté se mérite, d'où la nécessité de mesurer les résultats scolaires et, si nécessaire, de les sanctionner.

Il faut enseigner, par exemple, que le niveau et les conditions de vie en France, s'ils comptent parmi les meilleurs au monde, sont non pas le fruit du hasard, mais le résultat du travail, de la volonté et de la capacité d'innovation des générations qui nous ont précédés pour bâtir une République riche et solidaire. L'éducation doit donc enseigner qu'il est du devoir de chacun de prendre sa part dans l'effort collectif de la nation, de rechercher un emploi, de s'engager dans un cadre associatif, sportif, syndical ou politique..., pour faire vivre l'idéal de liberté, d'égalité et de fraternité. Il s'agit de mettre en œuvre les valeurs interpersonnelles en récusant l'individualisme forcené qui nuit au corps social tout entier. C'est ainsi que l'enseignement de la nation peut surmonter les difficultés nées des questionnements examinés ci-dessus. 